\title{
Electron Beam Magnetometer
}

\author{
L. Marton, Lewis B. Leder, J. W. Coleman, ${ }^{2}$ and D. C. Schubert
}

(April 28, 1959)

\begin{abstract}
A theoretical investigation of the electron optics of an electron beam deflection method for detecting small magnetic fields is presented. It is shown that remarkably high sensitivity can be reached. A laboratory model of such a magnetometer was constructed, and it was demonstrated that the theoretical estimate of sensitivity, $3 \times 10^{-5}$ ampere per oersted, could be attained in practice. A discussion of the possible improvements which could extend the sensitivity of the device is also given.
\end{abstract}

\section{Introduction}

The work to be described was performed in 1952, and at its conclusion a report for the sponsoring agency only was prepared $[1] .^{3}$ Since at present there appears to be revived interest in electron beam magnetometers, it was felt that a published account of our results would serve a useful purpose.

The electron beam magnetometer is based on the fact that an electron beam will be deflected by a magnetic field. However, to obtain optimum performance the electron optics of such a system must be considered in detail. In 1955 Cragg [2] reported on an electronic magnetometer which consisted essentially of a miniature cathode-ray tube. The magnetic field was measured by compensating the beam deflection with a voltage applied to deflecting plates. With such a system he was able to measure a field of $10^{-4}$ oersted. It will be shown below that the electron beam magnetometer can theoretically measure changes of field as small as $3 \times 10^{-8}$ oersted.

\section{Theoretical Considerations}

We will consider a system which consists of an electron gun, a long-focus lens and a detector, and will determine the minimum detectable field for a given geometry and operating conditions. If we assume that we have a line source of electrons of finite width and perpendicular to the axis, then the current distribution in the image at the detector after the electrons have passed through the lens would look as in figure 1 (dashed line). In the case of a point source of electrons, the figure represents a section along a diameter of the image. The ordinate is current density and the abscissa is distance across the image. When diffraction and lens aberration are present a decrease in current density from maximum to zero takes place at the edge of the image in a distance $2 r$ (solid line fig. 1).

If the detector consisted of two slits each of width $2 r$ and at a distance $R-r$ from the center of the image, one could then measure a difference in current as

1 This work was in part supported by the U.S. Office of Naval Research and the Office of Basic Instrumentation of the National Bureau of Standards.

${ }^{2}$ Now at RCA Laboratories, Camden, N.J.

3 Figures in brackets indicate the literature references at the end of this paper.

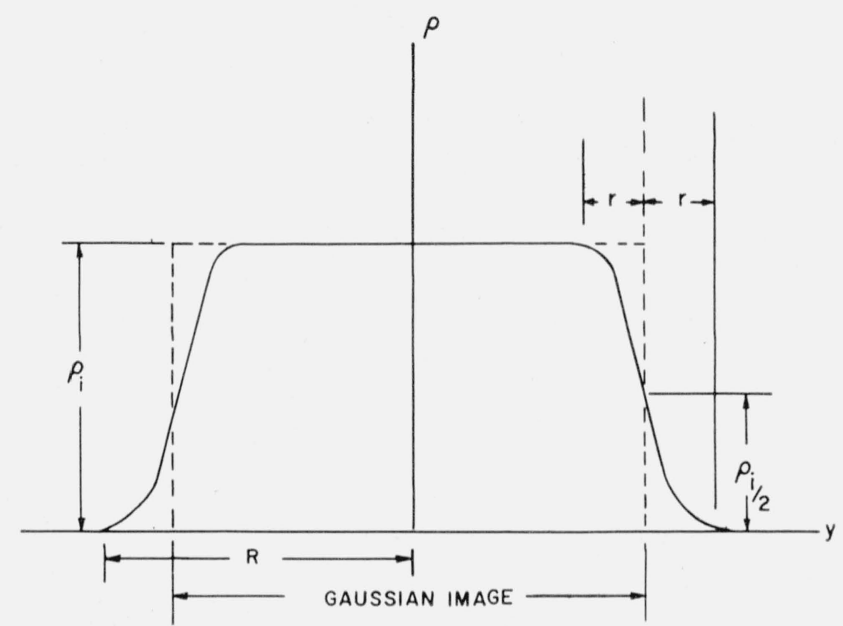

Figure 1. Cross section of electron beam at detector.

Dashed line is cross section without aberrations, and solid line with aberrations. $2 r$ is the aberration region.

the electron beam moved across the slits. Because the current density is changing so rapidly in the aberration region, a small motion of the beam would give a relatively large signal change. The minimum (min) detectable deflection would be

$$
y_{\mathrm{min}}=\frac{r i_{\mathrm{mln}}}{i_{0}}
$$

where $i_{0}$ is the maximum current difference when the image has moved so far that no current appears in one slit, and $i_{\min }$ is the minimum detectable current difference due to the movement $y_{\min }$ of the image.

The aberration region is due to three principal terms:

$$
\begin{aligned}
\text { spherical aberration, } r_{s} & \cong \frac{5 L^{3} \alpha^{3}}{(M+1)^{2} G d^{2}} \\
\text { chromatic aberration, } r_{c} & =\frac{2 L T \alpha}{1.16 \times 10^{4} V} \\
\text { diffraction, } r_{d} & =\frac{7.38 \times 10^{-8} M}{\alpha V^{1 / 2}}
\end{aligned}
$$

where $d$ is the effective lens thickness in centimeters, 
$G$ is a lens figure of merit, ${ }^{4} T$ is the absolute temperature of the emitter, $L$ is the total length of the beam path in centimeters, and $V$ is the energy of the electron beam in electron volts. In practice, the lower limit of the lens aperture will be set by the requirement that the maximum current density in the image must be

$$
\rho_{i}=\frac{i_{0}}{2 r l}
$$

where 1 is the length of the detector slit. If we assume that a ratio of 50 for $1 / r$ is a practical limit, this gives

$$
\rho_{i}=\frac{i_{0}}{100 r^{2}} .
$$

One can consider the crossover image formed by the electron gun as an effective object since the filament to crossover distance is, normally, small. This effective object then has a current density [3]

$$
\rho_{0}=\frac{e V \alpha^{2}}{300 k T} \rho_{f}
$$

in directions that will be intercepted by the long focus lens, where $\rho_{f}$ is the current density at the filament, $e$ is the charge of the electron, and $k$ is Boltzmann's constant. Then at the final image

$$
\rho_{i}=\frac{\rho_{0}}{M^{2}}=\frac{1.16 \times 10^{4} V \alpha^{2}}{M^{2} T} \rho_{f}=\frac{i_{0}}{100 r^{2}}
$$

Thus when other parameters have been chosen, $\alpha$ must be made large enough so that

$$
\alpha^{2} \gg \frac{M^{2} T i_{0}}{1.16 \times 10^{6} V r^{2} \rho_{f}}
$$

Since $r$ is the width of the aberration region, and in the worst case would be the sum of the absolute values of $r_{s}, r_{c}$, and $r_{d}$, one can substitute the sum of eq (3), (4) and (5) squared for $r^{2}$ in eq (9) and solve for $\alpha$.

If we now select reasonable parameters as follows: $V=1,000 \mathrm{v}, L=100 \mathrm{~cm}, M=1, T=3,000^{\circ} \mathrm{K}, i_{0}=1$ $\times 10^{-9} \mathrm{amp}, i_{\mathrm{min}}=4 \times 10^{-12} \mathrm{amp}, \rho_{f}=1 \mathrm{amp} / \mathrm{cm}^{2}, G$ $=0.2$, and $d=0.5 \mathrm{~cm}$, then

$$
\begin{aligned}
& \alpha=3.06 \times 10^{-4} \text { radians } \\
& r_{s}=1.43 \times 10^{-4} \mathrm{~cm} \\
& r_{c}=0.159 \times 10^{-4} \mathrm{~cm} \\
& r_{d}=0.036 \times 10^{-4} \mathrm{~cm}
\end{aligned}
$$

Therefore, $r$ becomes approximately $1.67 \times 10^{-4} \mathrm{~cm}$ (the sum of $r_{s}, r_{c}$, and $r_{d}$ ) so that from eq (1)

$$
y_{\min }=\frac{r i_{\min }}{i_{0}}=6.66 \times 10^{-7} \mathrm{~cm}
$$

\footnotetext{
4 Upper limits for $G$ are given; the optimum for a magnetic lens is $G=2.0$; for a unipotential electrostatic lens, $G=0.267$. Equation (3) is valid only when $d / L<<1$.
}

Now the deflection of an electron beam due to a magnetic field is given by

$$
y=\frac{b H L^{2}}{2 c} \sqrt{\frac{150 e}{m V}}=\frac{0.15 b H L^{2}}{V^{1 / 2}}
$$

where $H$ is the unknown magnetic field in oersted, $e$ the electronic charge in esu, $m$ the mass of the electron in grams, and $c$ the velocity of light in centimeters per second. The constant $b$ depends on the optics of the system as follows.

If the electron gun produces a beam which has no crossover or has a crossover in the strong field between cathode and anode, the beam will appear to diverge from a point behind the cathode. This is shown as the dashed trajectory on figure 2 . Then it can be shown that

$$
b=1-\frac{x_{1}^{2}\left(d+x_{1}+x_{3}\right)}{\left(x_{1}+x_{3}\right)^{2}\left(d+x_{1}\right)} .
$$

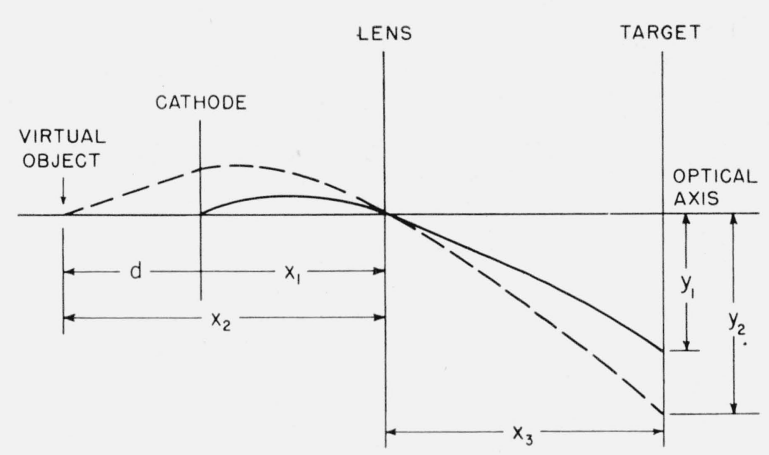

FIgURE 2. Electron trajectories for electron source at cathode and virtual object position.

If, however, the beam forms a crossover at the anode or a short distance in front of it, the beam can be considered as originating at the cathode since the cathode to anode distance is small compared to the total length of the electron beam path. In this case $d$ in eq (11a) is equal to zero and the beam would then follow a path as shown by the solid line in figure 2 . Then $b$ simplifies to

$$
b=1-\frac{x_{1}}{x_{1}+x_{3}} .
$$

Since $x_{3} / x_{1}$ is the magnification $M, b$ in eq (12b) turns out to be equal to $M / M+1$.

$L$ and $V$ can be considered constants of the system and therefore the deflection of the beam is proportional to the field. From this we determine, using eq (10), that $H_{\mathrm{min}}=2.8 \times 10^{-8}$ oersted. This is the minimum field which the device can detect when the selected parameters are used. In terms of current sensitivity this gives a value of $1.42 \times 10^{-9} \mathrm{amp} / \gamma$ (where $\gamma$ equals $10^{-5}$ oersted).

Since the width of the aberration region $r$ is approximately $1.67 \times 10^{-4} \mathrm{~cm}$, the proposed slit-system 
detector of $2 r$ by $50 r$ would be $3.34 \times 10^{-4} \mathrm{~cm}$ by $8.35 \times 10^{-3} \mathrm{~cm}$. Below, we consider a somewhat simpler system wherein the detector (fig. 3) consists of two plates overlapping in such a way that the image will, in balance, appear half on each plate.

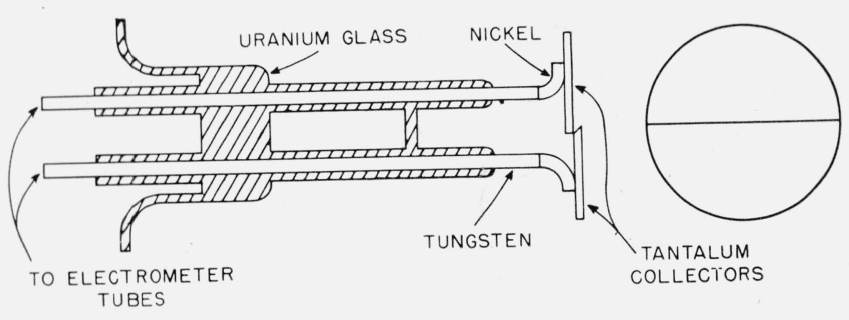

Figure 3. Collector plate assembly.

\section{Alternate Detecting System}

Whereas in the previous section the current density was considered in a region on the edge of the image, in this case we consider half the image as the sensing element, so that the current density will be

$$
\rho_{i}=2 i_{1} / \pi R^{2}
$$

where $i_{1}$ is the current due to half the image appearing on one collector plate and $R$ is the radius of the image. Then

$$
i_{\mathrm{min}}=4 y_{\mathrm{min}} R \rho_{i}
$$

where $y_{\mathrm{min}} \ll R$, or

$$
i_{\mathrm{min}}=8 i_{1} y_{\mathrm{min}} / \pi R .
$$

For this system we now select parameters: $V=$ $1,000 \mathrm{v}, L=100 \mathrm{~cm}, M=1, T=3,000^{\circ} \mathrm{K}, i_{1}=1 \times 10^{-8}$ amp, $R=0.01 \mathrm{~cm}$. We retain the minimum field $H=2.8 \times 10^{-8}$ oersted for a deflection $y=6.66 \times 10^{-7}$ $\mathrm{cm}$ and find the minimum current to be

$$
i_{\min }=\frac{(8)\left(1 \times 10^{-8}\right)\left(6.66 \times 10^{-7}\right)}{(3.14)(0.01)}=1.7 \times 10^{-12} \mathrm{amp} .
$$

For the same expected minimum field as in the first case we now have a sensitivity of $6 \times 10^{-10} \mathrm{amp} / \gamma$. This is 0.42 times the signal in the first case, but, as will be pointed out later, reasonable changes can be made in $i_{1}$ and $R$ to bring the minimum current output to a much larger value.

\section{Sources of Noise}

The above calculations are made on the assumption that no interference or noise exist. Since the smallest detectable signal is ultimately determined by the magnitude of interfering signals, it is necessary to form some idea of what such limitations may be.
1. External interference can be due either to (a) time fluctuations in the ambient field, or (b) geologic anomalies (i.e., nonuniformities in the earth's field caused by variations in the magnetic properties of the earth's crust). For the first fluctuation Fromm [4] has found that in the frequency range 0.1 to $2 \mathrm{cps}$ the noise does not exceed $0.03 \gamma\left(3 \times 10^{-7}\right.$ oersted $)$ for more than 50 percent of the time. It is believed that a gradient system of measurement, wherein this fluctuation is cancelled out, can be used successfully with a stationary magnetometer. This will be discussed later. Signal (b) occurs if the magnetometer is in motion so that for a fixed instrument, which we are considering, this can be ignored.

2. Internal or instrumental noise can be caused by several factors which will be investigated individually. While vibration can cause considerable noise, this is merely a question of rigidity of construction and mounting which can be overcome successfully.

The other sources of noise occur in the electron gun and in the input stage of the measuring circuit. They are (a) resistor (thermal) noise in the input resistor, (b) flicker noise in the detector tube, (c) grid current of the detector tube, and (d) shot noise in the magnetometer tube.

(a) Resistor noise is given by [5]

$$
\overline{E_{R}^{2}}=4 k T R B
$$

where $R$ is the resistance in ohms and $B$ is the bandwidth in cycles per second, $k$ is Boltzmann's constant and $T$ the absolute temperature. With $\mathrm{R}=10^{9} \mathrm{ohms}, B=1 \mathrm{cps}$ and $T=300^{\circ} \mathrm{K}$, the $\mathrm{rms}$ value of $E_{R}=3.98 \mu \mathrm{v}$.

(b) Flicker noise in the detector tube is given by O'Meara [6] for electrometer tubes as being between $400 \mu \mathrm{v}$ and $900 \mu \mathrm{v}$ for randomly selected tubes, and $200 \mu \mathrm{v}$ for compensated circuits. With careful selection of tubes the electrometer may be operated down to the limit of sensitivity set by the thermal grid noise.

(c) Tubes are available and can be selected whose thermal grid noise (grid current) is $10^{-14}$ amp or less.

(d) Shot noise in the magnetometer arises in the electron gun. According to Haine and Mulway [7] the type of gun used can be considered as a temperature limited diode, so that the noise current would be given by [5]

$$
i_{s}=\sqrt{2 e i_{1} B} \text { amps }
$$

where $i_{1}$ is the emission current, $B$ was defined previously, and $e$ is the charge of the electron. Then for the alternate detection system

$i_{s}=\sqrt{(2)\left(1.6 \times 10^{-19}\right)\left(4 \times 10^{-8}\right)(1)}=1.13 \times 10^{-13} \mathrm{amps}$

The first three types of noise, (a), (b), and (c), will not present any serious problem, since with the minimum signal current of $1.7 \times 10^{-12}$ amps imposed on a $10^{9}$-ohm resistor we will have a signal of 1,700 $\mu \mathrm{v}$. For the worst case of flicker noise this should 
still give a signal-to-noise ratio of 2 and in the best case approximately 8.5. With the parameters previously chosen, the shot noise turns out to be approximately 0.1 the minimum signal, giving an expected signal-to-noise ratio of approximately 10 .

\section{Experimental Design}

The basic design consisted of an electron gun, electrostatic lens, and overlapping collector plates enclosed in a glass envelope which could be evacuated and sealed off from the pumping system. The metal parts were made either of tantalum or nonmagnetic stainless steel, and the spacers were made of glass and porcelain tubing. The electrical connections were made through tungsten wire presses at each end of the glass envelope. The outer surface of the glass envelope was painted with aquadag and connected to ground to reduce the effects of surface leakage and stray capacitances. Figure 4 is a drawing of the assembly.

The electron gun was patterned after one described by Steigerwald [8], and has the virtue of having a long crossover distance which can be controlled over a wide range by varying the bias voltage. The dimensions are given in figure 5 .

The electrostatic lens was a symmetrical three electrode system consisting of two identical apertured disks at ground potential with a central apertured disk at negative potential. When oper.ated $50 \mathrm{v}$ below the accelerating potential, the lens had a focal length of $18 \mathrm{~cm}$. The collector plates were placed at $30 \mathrm{~cm}$ from the center of the lens. Therefore, to obtain an image on the collector plates, it is found, using the thin lens formula of light optics, that the object distance must be $45 \mathrm{~cm}$. Since the distance from the cathode to the lens was fixed at $25 \mathrm{~cm}$, the beam was made to diverge from a virtual source $20 \mathrm{~cm}$ behind the cathode. Under these conditions $d$ (on fig. 2) is $20 \mathrm{~cm}$, and from eq (11a) $b$ is found to be 0.66 .

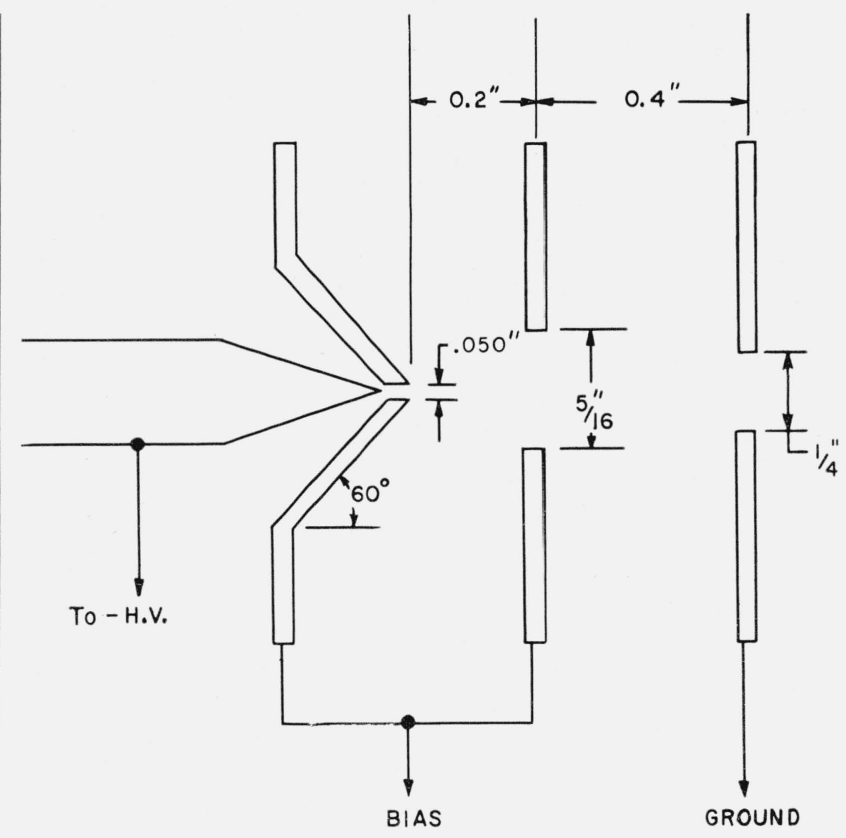

Figure 5. Dimensions in inches for Steigerwald-type electron gun used.

We have now two changes from the postulated case which will require recalculating the expected current change for a signal. One change is that due to the shift of the object position, and the other is that due to the shorter path $(55 \mathrm{~cm}$ instead of 100 $\mathrm{cm})$.

If we consider $2.8 \times 10^{-8}$ oersted (the $H$ calculated for the first case) as the minimum signal we wish to measure, we now get for $y_{\min }$, from eq (11),

$$
y_{\min }=\frac{(0.15)(0.66)\left(2.8 \times 10^{-8}\right)(55)^{2}}{\sqrt{1,000}}=2.61 \times 10^{-7} \mathrm{~cm}
$$

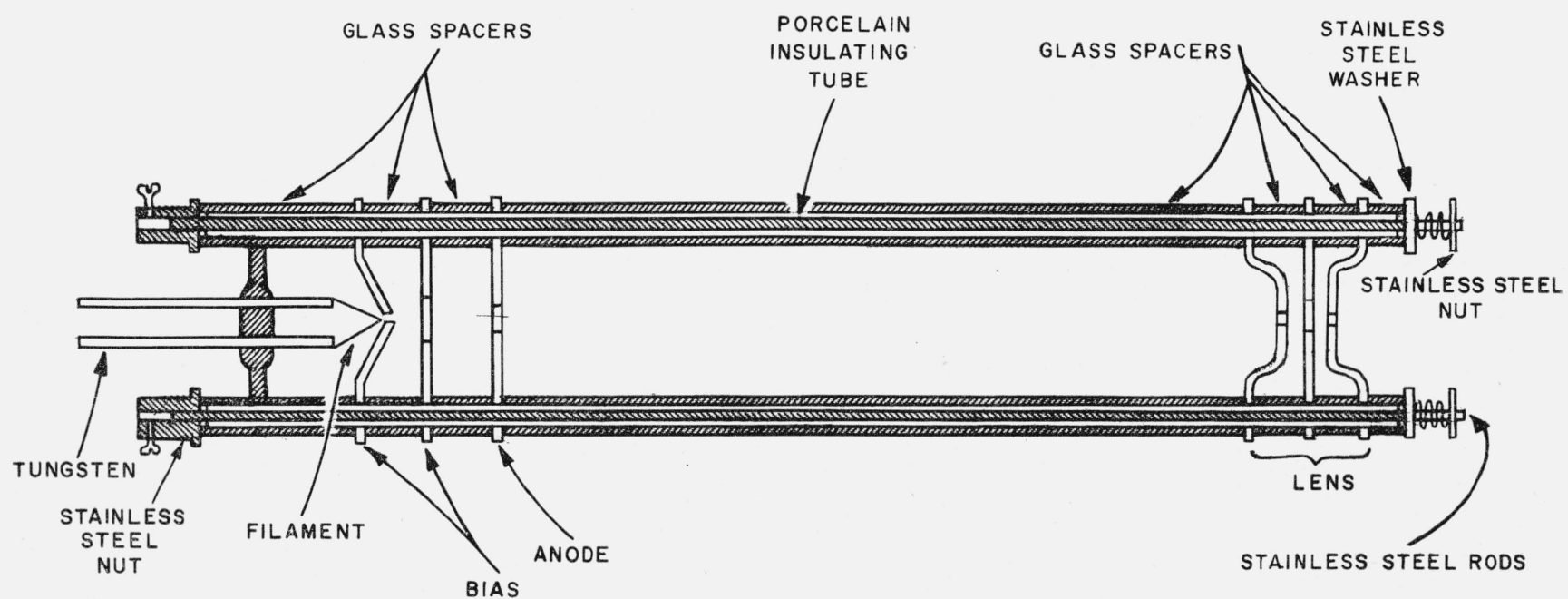

Figure 4. Electron gun and electrostatic lens assembly. 
and $i_{\min }$ from eq $(15)$ is

$$
i_{\min }=\frac{(4)\left(2 \times 10^{-8}\right)\left(2.61 \times 10^{-7}\right)}{(3.14)(0.01)}=6.65 \times 10^{-13} \mathrm{amp} .
$$

This corresponds to an expected sensitivity of $2.4 \times 10^{-10} \mathrm{amp} / \gamma$ for $1,000-\mathrm{V}$ acceleration and $2.9 \times 10^{-10} \mathrm{amp} / \gamma$ for $700 \mathrm{v}$.

The measuring circuit was required to measure a beam current of the order of $4 \times 10^{-8}$ amp and, more important, measure differences of the order of $10^{-12}$ amp or less. The first part, that of measuring the total current, was taken care of by using a millivoltmeter with shunt box which could measure down to $10^{-11}$ amp. The second, that of measuring a difference of 1 part in 10,000 between two d-c signals at a level of $10^{-8}$ amps required considerable design effort since no suitable low-level differential amplifiers were available at the time. A satisfactory solution was arrived at by using a modified version of the Nier mass-ratio measuring method [9]. More elegant solutions to this problem are now possible.

Since the most sensitive stage of this differential amplifier was the input tube, the two electrometer tubes and their two hi-meg grid resistors were soldered directly to the collector-plate leads of the magnetometer tube, and the connections to the rest of the amplifier were made with long leads so that the amplifier could be kept at some distance from the magnetometer tube. The electrometer tube assembly was enclosed in a glass "bottle" which was waxed to the end of the magnetometer tube. A stopcock on the bottle made it possible to evacuate it and then seal it off. The bottle was painted with aquadag and grounded to keep the tube light tight and reduce the effect of external capacitances.

The final unit in the magnetometer tube was the collector plate assembly. This was made up of two semicircular phosphor-coated plates overlapping along the straight edges, but electrically separated. They were alined at $90^{\circ}$ to the electron beam. It was highly important that these be rigidly mounted since vibration could cause a considerable noise signal. The construction is shown in figure 3 . It was also highly important that the resistance between these two plates and between each plate and ground be high compared to the input resistance to the electrometer circuit. A measurement of this gave a value of $1.7 \times 10^{13} \mathrm{ohms}$.

The circuit for the magnetometer tube is shown on figure 6 . The filament was heated with a-c using a variac and transformer. The transformer was specially built with a $2.5-\mathrm{v}$ secondary and insulation for $5,000 \mathrm{v}$. The electron gun was self-biased as shown. A divider network was placed across the high-voltage supply, and the lens voltage was obtained from this by inserting a banana-plug connection at the position giving the best definition of image. The high-voltage supply consisted of a commercial 5 -kv supply with 5 percent regulation and a series tube regulator circuit to bring the stability to 1 part in 13,000 .

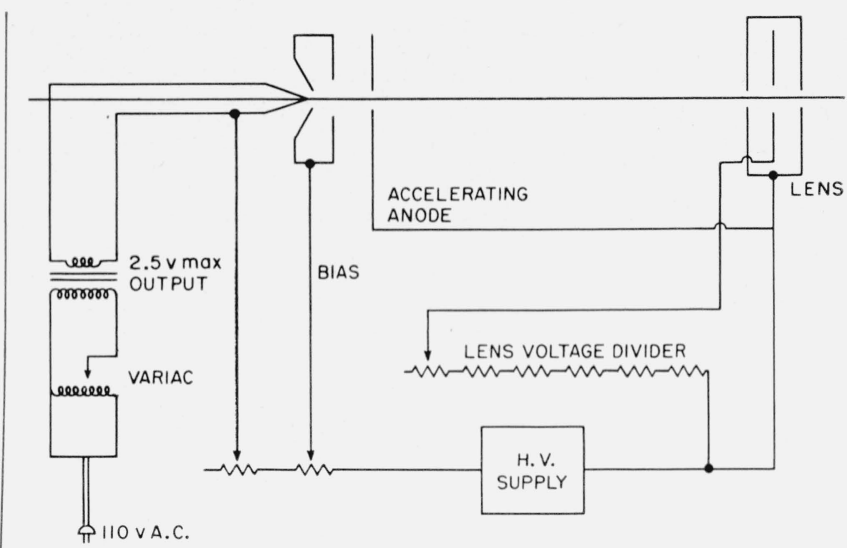

Figure 6. Electrical connections to electron gun and lens.

The magnetometer was placed at the center of a set of four rectangular coils (with each pair in series) to compensate for the horizontal and vertical components of the earth's magnetic field [10].

\section{Experimental Tests}

To test the operation of the detector the amplifiers were balanced to give a zero difference with the electron beam off. With the beam on, it was found that zero balance was obtained when the beam was all on one plate and just barely touching the other. Figure 7 shows the current readings on each plate as the beam was moved across the dividing edge by changing the coil current. It was felt that this effect was probably due to ion currents and charging of the glass surface. To correct this a 1-in. wide aquadag ring was printed on the inside of the glass tube $1 / 8$ in. from the collector plates, and a tungsten wire was sealed through the glass so that it was even with the inner surface and making contact with the aquadag. It was found that when this guard ring was grounded or a positive or nega-
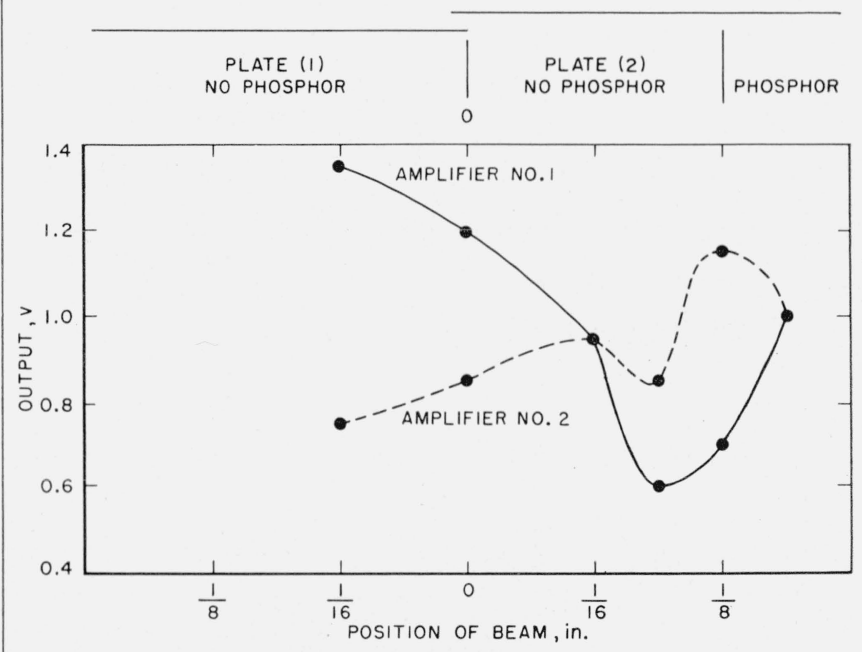

Figure 7. Amplifier-output signal versus beam position relative to detector edge. 
tive voltage was applied the beam could be balanced with what visually appeared to be half the beam on each plate. Figure 8 shows the change in output per plate as the voltage on the guard ring was varied from positive to negative. The position of the beam was adjusted to be half on each plate. Figure 9 shows the current on each plate as the beam was moved across the edge with the guard ring at ground potential and at $-5 \mathrm{v}$ and $+5 \mathrm{v}$. It is seen that with the guard ring at ground the beam balances on the edge, but as the beam moves more on to one plate the polarity changes. With either a negative or a positive voltage on the guard

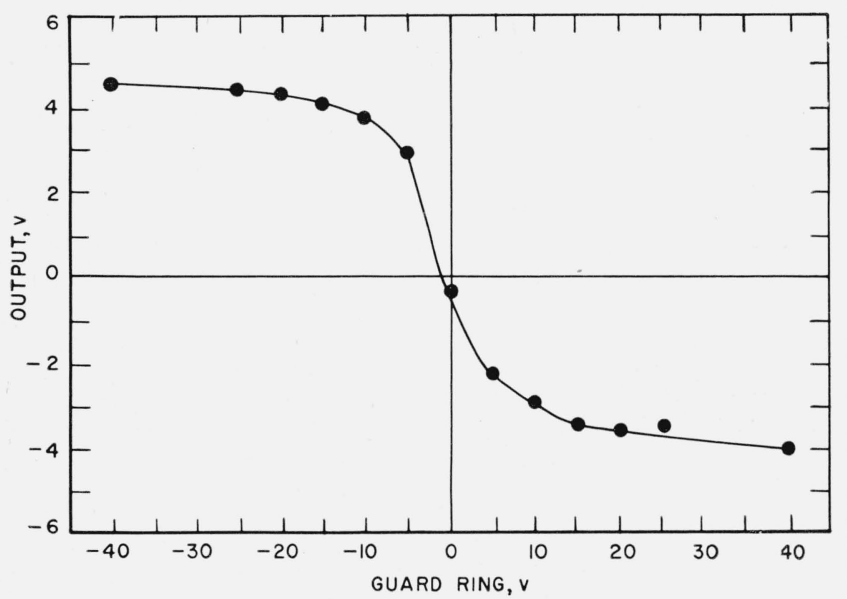

Figure 8. Output signal as a function of guard-ring voltage. The electron beam was half on each detector plate.

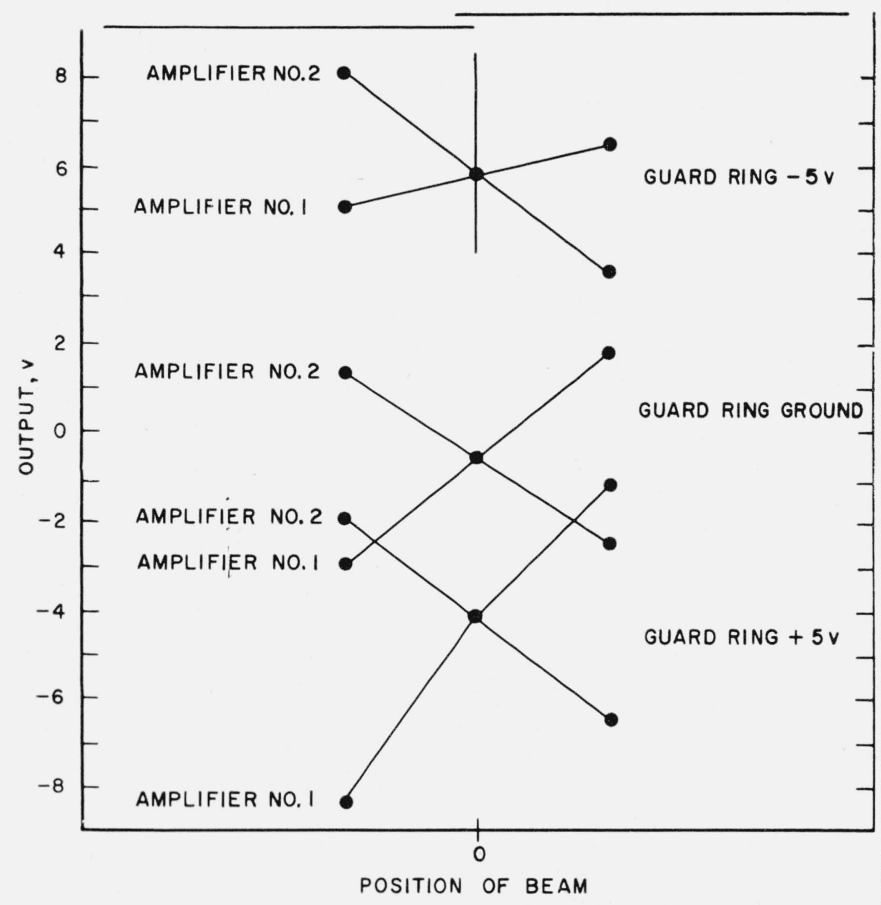

Figure 9. Amplifier-output signal versus beam position for three guard-ring potentials. ring the output was zero for balance position and did not change sign as the beam was moved across the edge.

When the beam was centered, the output signal showed a great deal of fluctuation. This, however, appeared to correlate with the movement of automobiles at a distance of about $200 \mathrm{yd}$ from the building in which the tests were being made. To overcome this the entire equipment was moved to Blossom Point Proving Grounds, Md., ${ }^{5}$ and set up in a shack located 5 miles from where other tests were being made. The location chosen was peninsula-shaped and on the land side the nearest building was over 1 mile away. The compensating coils and tube were set up in approximately north-south orientation with the measuring edge of the collector plates in a horizontal position.

\section{Experimental Results}

The sensitivity was tested by turning on and off a calibrated coil. The coil had an average diameter of $15 \mathrm{~cm}$ and produced a field of $11 \mathrm{\gamma} / \mathrm{amp}$ at a distance of $200 \mathrm{~cm}$. Three measurements of sensitivity and stability were made with the center of the coil placed at a distance of $200 \mathrm{~cm}$ from the center of the magnetometer tube. The axis of the coil was perpendicular to the tube, parallel to the edges of the collector plates, and midway between the lens and collector plates.

Table 1 gives the results of these three tests. In every case the filament was operated at $1.1 \mathrm{v}$ a-c using a constant voltage transformer. The guard ring was at ground potentiz.. In the second and third tests a laboratory built 2-kv power supply replaced the commercial 5-kv supply used for the accelerating voltage, the bias was increased and the beam recentered. In the third case the accelerating voltage was reduced from $1000 \mathrm{v}$ to $700 \mathrm{v}$ d-c.

TABLE 1

\begin{tabular}{|c|c|c|c|}
\hline & Test 1 & Test 2 & Test 3 \\
\hline $\begin{array}{l}\text { A ccelerating voltage } \\
\text { Test field, } H_{d} \\
\text { Avg change output. } \\
\text { Avg change output.-. } \\
\text { Experimental sensitivity_amp } / \gamma_{--}\end{array}$ & $\begin{array}{c}1000 \\
0.74 \\
56 \\
0.56 \times 10^{-10} \\
0.76 \times 10^{-10}\end{array}$ & $\begin{array}{c}1000 \\
0.80 \\
108 \\
\text { 1. } 08 \times 10^{-10} \\
\text { 1. } 36 \times 10^{-10}\end{array}$ & $\begin{array}{c}\quad 700 \\
0.80 \\
251 \\
2.51 \times 10^{-10} \\
3.15 \times 10^{-10}\end{array}$ \\
\hline 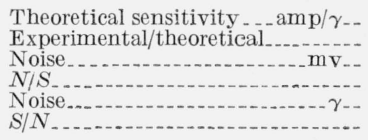 & $\begin{array}{c}2.4 \times 10^{-10} \\
0.3 \\
21 \\
0.375 \\
0.278 \\
2.67\end{array}$ & $\begin{array}{c}2.4 \times 10^{-10} \\
0.6 \\
16 \\
0.148 \\
0.118 \\
6.75\end{array}$ & $\begin{array}{l}2.9 \times 10^{-10} \\
1 \\
26 \\
0.103 \\
0.086 \\
9.7\end{array}$ \\
\hline
\end{tabular}

The increased sensitivity in the second test was probably the result of increased current density due to increasing the bias. The noise level given above included both instrumental noise and short-term magnetic field variations. A decrease in instrumental noise may have taken place due to the use of a different power supply, but this was not definitely

${ }^{5}$ The Blossom Point Proving Ground, Md., was operated by the Ordnance Development Division of the NBS, which is now the Diamond Ordnance Fuse Laboratories operated by the Dept. of the Army. 
established. The improvement in sensitivity in the third case was probably due to the decreased accelerating potential (since the deflection varies as the inverse square root of the accelerating voltage) and increased bias which apparently reduced the spot size.

The experimental to theoretical ratios of $0.3,0.6$, and 1 indicate that the theoretical approach was reasonably accurate, and that even greater sensitivities can be obtained. The discrepancy probably lies in the original estimate of the radius of the image, $0.01 \mathrm{~cm}$, which was selected as a reasonably attainable image size. It would appear from the test results that in the first two cases the spot size was larger than this value, but approximately equal to it in the last case.

\section{Conclusions and Suggestions for Improvement}

While it was shown that the expected sensitivity was closely approached, further improvements could be made. These would involve increasing the length of the tube, decreasing the spot size, and decreasing the accelerating potential.

If we select parameters $V=600 \mathrm{v}, L=100 \mathrm{~cm}$, $M=1, T=3,000^{\circ} \mathrm{K}, i_{i}=1 \times 10^{-8}, R=0.05 \mathrm{~mm}$, and $\mathrm{H}=2.8 \times 10^{8}$ oersted, then from eq (11) we would expect a minimum deflection

$$
y_{\min }=\frac{(0.15)\left(2.8 \times 10^{-8}\right)(100)^{2}}{2 \sqrt{600}}=0.85 \times 10^{-6} \mathrm{~cm}
$$

and from eq (15) a minimum signal

$$
i_{\mathrm{min}}=\frac{(4)\left(1 \times 10^{-8}\right)\left(0.85 \times 10^{-6}\right)}{(3.14)(0.005)}=2.17 \times 10^{-12} \mathrm{amp}
$$

corresponding to a sensitivity of $7.83 \times 10^{-10} \mathrm{amp} / \gamma$.

Another way to obtain increased sensitivity would be to use field emission. ${ }^{6}$ Current densities at the cathode as high as $6 \times 10^{9} \mathrm{amp} / \mathrm{cm}^{2}$ have been obtained, and values of the order of $10^{7} \mathrm{amp} / \mathrm{cm}^{2}$ are fairly easily obtained. Since the previous calculation calls for only $0.131 \mathrm{amp} / \mathrm{cm}^{2}$, it is seen that with field emission in place of thermionic emission one can easily obtain a set of conditions to give considerably greater sensitivity with the present basic design of tube.

${ }_{6}^{6}$ This was not tried at the time due to the more difficult problem involved and the limited time available.
It is also possible that improvement in image characteristics could be obtained by replacing the electrostatic lens with an aperture and external coil for focusing the beam. This system would have less aberration and, therefore, give a more concentrated image.

In a stationary system one could eliminate the ambient field fluctuation by using a gradiometer arrangement. This would consist of two magnetometer tubes at some convenient distance apart, each surrounded by a set of field compensation coils. A deflection due to a magnetic field change produces a signal in tube 1 , which is used to excite both sets of coils. This is done in such a way, using a servosystem, as to bring the beam in tube 1 back to zero. If a gradient exists between tube 1 and tube 2 , the feedback will not bring the beam in tube 2 back to zero. This signal would then be an indication of the field gradient over the base distance between the tubes.

The authors acknowledge the assistance of J. A. Simpson for discussions and work done on the theoretical considerations and circuitry, J. J. Madine for the glass work, and L. Mann and A. Dauses for the machine work. The authors also thank the Ordnance Development Division of the National Bureau of Standards (now Diamond Ordnance Fuse Laboratory, U.S. Army) for making available the test location at Blossom Point, Md.

\section{References}

[1] L. Marton, L. B. Leder, and J. W. Coleman (private communication).

[2] B. G. Cragg, J. Sci. Instr. 32, 385 (1955).

[3] D. B. Langmuir, Proc. IRE 25, 977 (1937).

[4] W. Fromm, Advances in Electronics, 4, 259 (Academic Press, New York, N.Y., 1952).

[5] S. Goldman, Frequency analysis, modulation and noise, pp. 362, 392 (McGraw-Hill Book Co., Inc., New York, N.Y., 1938).

[6] F. E. O’Meara, Rev. Sci. Instr. 22, 106 (1951).

[7] M. E. Haine \& T. Mulway, Electron Physics Symposium, Washington, D.C. (Nov. 1951).

[8] K. H. Steigerwald, Optik 6, 469 (1949).

[9] A. O. Nier, E. P. Ney \& M. G. Ingraham, Rev. Sci. Instr. 18, 294 (1947).

[10] R. H. Lyddane \& A. E. Ruark, Rev. Sci. Instr. 10, 253 (1939).

Washington, D.C.

(Paper 63C1-8) 